\title{
RADIAL FUNCTIONS AND INVARIANT CONVOLUTION OPERATORS
}

BY

\author{
CHRISTOPHER MEANEY
}

\begin{abstract}
For $1<p<2$ and $n>1$, let $A_{p}\left(\mathbf{R}^{n}\right)$ denote the FigàTalamanca-Herz algebra, consisting of functions of the form

$$
\sum_{k=0}^{\infty} f_{k} * g_{k}
$$

with $\sum_{k}\left\|f_{k}\right\|_{p} \cdot\left\|g_{k}\right\|_{p^{\prime}}<\infty$. We show that if $2 n /(n+1)<p<2$, then the subalgebra of radial functions in $A_{p}\left(R^{n}\right)$ is strictly larger than the subspace of functions with expansions (*) subject to the additional condition that $f_{k}$ and $g_{k}$ are radial for all $k$. This is a partial answer to a question of Eymard and is a consequence of results of Herz and Fefferman. We arrive at the statement above after examining a more abstract situation. Namely, we fix $G \in[F I A]_{B}$ and consider ${ }^{B} A_{p}(G)$ the subalgebra of $B$-invariant elements of $A_{p}(G)$. In particular, we show that the dual of ${ }^{B} A_{p}(G)$ is equal to the space of bounded, right-translation invariant operators on $L^{p}(G)$ which commute with the action of $B$.
\end{abstract}

Introduction. In his survey of the properties of the Figà-Talamanca-Herz algebras $A_{p}(G)$, Eymard asks the following question, [Ey, 9.3]. If $u \in A_{p}\left(\mathbf{R}^{n}\right)$ is radial does it have an expansion

$$
u=\sum_{l=0}^{\infty} f_{l} * g_{l}
$$

with not only the usual conditions $f_{l} \in L^{p}\left(\mathbf{R}^{n}\right), g_{l} \in L^{p^{\prime}}\left(\mathbf{R}^{n}\right)$, and

$$
\sum_{l=0}^{\infty}\left\|f_{l}\right\|_{p}\left\|g_{l}\right\|_{p^{\prime}}<\infty
$$

but also $f_{l}$ and $g_{l}$ radial for all $l$ ?

We use results of Herz and Fefferman to show that the answer is no when $n>1$ and $2 n /(n+1)<p<2$.

A similar statement is possible for central functions in $A_{p}(G)$, where $G$ is a compact, simply connected, simple Lie group.

It is possible to view the radial part of $A_{p}\left(\mathbf{R}^{n}\right)$ in a more general setting. Suppose that $G$ is a locally compact group with a group of topological automorphisms $B$ such that $B$ contains all inner automorphisms of $G$ and $B$ is compact in $\operatorname{Aut}(G)$. We examine the subalgebra of $B$-invariant elements of $A_{p}(G)$, written ${ }^{B} A_{p}(G)$, and

Received by the editors April 20, 1983.

1980 Mathematics Subject Classification. Primary 43A15, 43A22, 42B15; Secondary 22D15, 43A75.

Key wonds and phrases. Radial function; Figà-Talamanca-Herz algebra, $[F I A]_{B}^{-}$, B-characters, convolution operator, compact semisimple Lie group, central function, multiplier, Fourier transform. 
show that its dual is the space of bounded right-translation invariant operators on $L^{p}(G)$ which commute with the action of $B$. Furthermore, let $\mathfrak{A}_{p}(G, B)$ be the image of $\left({ }^{B} L^{p}(G)\right) \hat{\otimes}\left({ }^{B} L^{p^{\prime}}(G)\right)$ under the map $f \otimes g \mapsto g * f^{\vee}$. For $h \in L^{1}(G)$, denote by $\lambda(h)$ the operator $f \mapsto h * f$, acting on $L^{p}(G)$ and having norm $\||\lambda(h)|\|_{p}$. If $h \in{ }^{B} L^{1}(G)$ then $\lambda(h):{ }^{B} L^{p}(G) \rightarrow{ }^{B} L^{p}(G)$ and we denote the norm of this operator by $N_{p}(f)$.

We arrive at our answer to Eymard's question via the following general principle. If there exists a sequence $\left\{h_{n}\right\}_{n} \in{ }^{B} L^{1}(G)$ with $\left\{||\left|\lambda\left(h_{n}\right)\right| \|_{p}\right\}_{n}$ unbounded and $\left\{N_{p}\left(h_{n}\right)\right\}_{n}$ bounded, then

$$
{ }^{B} A_{p}(G) \neq \mathfrak{A}_{p}(G, B)
$$

1. $[F I A]_{B}^{-}$groups. If $G$ is a locally compact group let $\operatorname{Aut}(G)$ be the group of topological automorphisms of $G$, equipped with the Birkhoff topology described in $[\mathbf{B r}]$ and $[\mathbf{P t S u}]$. Throughout this paper we assume that there is a subgroup $B \subset \operatorname{Aut}(G)$ such that: (i) $B$ contains all inner automorphisms of $G$; and (ii) $B$ is compact in $\operatorname{Aut}(G)$. This is abbreviated by writing $G \in[F I A]_{B}^{-}$. For a list of the properties of the class $[F I A]_{B}^{-}$see the survey article $[\mathbf{P a}]$.

Examples include locally compact abelian groups, with $B$ trivial; central groups, with $B$ equal to the group of inner automorphisms; and $G=\mathbf{R}^{n}, B=S O(n)$.

For $\alpha \in \operatorname{Aut}(G)$ and $f$ a function on $G$ we let ${ }^{\alpha} f(x)=f\left(\alpha^{-1}(x)\right), x \in G$. The hypothesis $G \in[F I A]_{B}^{-}$implies that $G$ is unimodular and we fix a Haar measure $m_{G}$ on $G$. In particular, $m_{G}$ is $B$-invariant (see [Br, §IV.5]). The action of $B$ extends to the Lebesgue spaces of $G$ with respect to $m_{G}$. If $f$ belongs to one of the spaces $L^{p}(G)(1 \leq p<\infty)$ or $C_{0}(G)$ then the map $\alpha \mapsto^{\alpha} f$ provides a strongly continuous representation of $B$ by isometries $[\mathbf{B r}$, p. 78]. If $E$ is a Lebesgue space or a space of functions on $G$ then we let

$$
{ }^{B} E:=\left\{f \in E:{ }^{\alpha} f=f, \forall \alpha \in B\right\} .
$$

Having equipped $G$ with $m_{G}$ we define convolution of functions on $G$ as in [HwRs, §20]. Since $m_{G}$ is $B$-invariant we see that

$$
{ }^{\alpha}(\varphi * \psi)=\left({ }^{\alpha} \varphi\right) *\left({ }^{\alpha} \psi\right), \quad \forall \alpha \in B,
$$

whenever $\varphi * \psi$ makes sense. Note also that

$$
\left({ }^{\alpha} f\right)^{\vee}={ }^{\alpha}\left(f^{\vee}\right), \quad \forall \alpha \in \operatorname{Aut}(G),
$$

where $f^{\vee}(x)=f\left(x^{-1}\right)$.

If $f \in C_{0}(G)$ or $L^{p}(G)(1 \leq p<\infty)$ we set

$$
Z_{B} f:=\int_{B}\left({ }^{\beta} f\right) d m_{B}(\beta),
$$

where the right-hand side is the Bochner integral with respect to the normalized Haar measure $m_{B}$ of the compact group $B$. This is denoted $f^{\#}$ in [Msk]. The operator $Z_{B}$ is obviously bounded and provides the projections $C_{0}(G) \rightarrow{ }^{B} C_{0}(G)$ and $L^{p}(G) \rightarrow{ }^{B} L^{p}(G)$. Since $B$ contains all inner automorphisms of $G,{ }^{B} L^{1}(G)$ is contained in the centre of $L^{1}(G)$.

The maximal ideal space of the commutative Banach algebra ${ }^{B} L^{1}(G)$ is identified with $\mathfrak{X}_{B}$, the space of $B$-characters as defined in [Msk, $\S 2$ ]. These can be considered as the zonal spherical functions for the $\mathrm{Gel}^{\prime}$ fand pair $(G \rtimes B,\{1\} \times B)$. Hence, $\mathfrak{X}_{B}$ 
can be equipped with a measure $\nu$ so that the Gel' fand transform $\mathcal{F}^{B}{ }^{B} L^{1}(G) \rightarrow$ $C_{0}\left(\mathfrak{X}_{B}\right)$ extends to an isometric isomorphism $\mathcal{F}^{B} L^{2}(G) \rightarrow L^{2}\left(\mathfrak{X}_{B}, \nu\right)$, see [Go]. The usual interpolation argument shows that if $1<p<2$ and $(1 / p)+\left(1 / p^{\prime}\right)=1$ then $₹$ extends to be a bounded map

$$
\text { ₹: }{ }^{B} L^{p}(G) \rightarrow L^{p^{\prime}}\left(\mathfrak{X}_{B}, \nu\right) .
$$

For further details on analysis on $[F I A]_{B}^{-}$groups see $[\mathrm{Ha}, \mathrm{HHL}, \mathrm{KS}, \mathrm{LM}, \mathrm{Mz}$, Msk, Pa, Pt, PtSu].

2. Figà-Talamanca-Herz spaces. Fix $G \in[F I A]_{B}^{-}$and $1<p<\infty$. The action of $G$ on $L^{p}(G)$ by right translation is denoted by

$$
(\rho(x) f)(y):=f(y x), \quad \forall x, y \in G,
$$

and left translation is

$$
(\lambda(x) f)(y):=f\left(x^{-1} y\right), \quad \forall x, y \in G .
$$

Furthermore, if $h \in L^{1}(G)$ and $f \in L^{p}(G)$ then we set

$$
\lambda(h) f:=h * f,
$$

so that $\lambda(h)$ is a bounded linear operator on $L^{p}(G)$. The space of all bounded linear operators on $L^{p}(G)$ which commute with $\rho(G)$ is denoted $C v_{p}(G)$ and is equipped with the operator norm $\|\cdot\| \|_{p}$. Clearly $\lambda: L^{1}(G) \rightarrow C v_{p}(G)$ is a homomorphism of Banach algebras.

The Figà-Talamanca-Herz space $A_{p}(G)$ is the image of $L^{p}(G) \hat{\otimes} L^{p^{\prime}}(G)$ under the map

$$
P(f \otimes g):=g * f^{\vee}
$$

and the norm on $A_{p}(G)$ is the quotient norm on $\left(L^{p}(G) \hat{\otimes} L^{p^{\prime}}(G)\right) / \operatorname{ker}(P)$. Since $G$ is amenable (see $\left[\mathbf{P a}\right.$, diagram 1]) we can identify $A_{p}(G)^{*}$ with $C v_{p}(G)$. For $T \in C v_{p}(G)$ and $\varphi \in A_{p}(G)$, having series expansion $\varphi=P\left(\sum_{n=0}^{\infty} f_{n} \otimes g_{n}\right)$, the pairing is

$$
\langle T, \varphi\rangle=\sum_{n=0}^{\infty} g_{n} *\left(T f_{n}\right)^{\vee}(1) .
$$

In particular, if $h \in L^{1}(G)$ then

$$
\langle\lambda(h), \varphi\rangle=\int_{J_{G}} \varphi h d m_{G} .
$$

Herz has shown that $C v_{p}(G)$ is the ultrastrong closure of $\lambda\left(C_{c}(G)\right)[\mathbf{H z} \mathbf{3}$, Theorem 5].

2.4 LEMMA. If $T \in C v_{p}(G)$ then there is a net $\left\{h_{\gamma}\right\}_{\gamma} \subset C_{c}(G)$ such that

$$
\left\|\left|\lambda\left(h_{\gamma}\right)\right|\right\|_{p} \leq\||| T \mid\|_{p}, \quad \forall \gamma
$$

and

$$
\langle T, \varphi\rangle=\lim _{\gamma} \int_{G} \varphi h_{\gamma} d m_{G}, \quad \forall \varphi \in A_{p}(G) .
$$

For details see [Cw, Ey, FT, Hz 2, Hz 3, Rb]. 
Herz also considered the map $M$, which takes functions on $G$ to functions on $G \times G$ and is defined by

$$
(M h)(x, y):=h\left(x y^{-1}\right), \quad \forall x, y \in G .
$$

In particular, if $\varphi \in A_{p}(G)$ then $M \varphi$ is a multiplier of $L^{p}(G) \hat{\otimes} L^{p^{\prime}}(G)$ and

$$
P\left((M \varphi) \sum_{n=0}^{\infty} f_{n} \otimes g_{n}\right)=\varphi \cdot P\left(\sum_{n=0}^{\infty} f_{n} \otimes g_{n}\right) .
$$

This shows that $A_{p}(G)$ is a Banach algebra (see [Ey, Théorème 1]).

We next consider the action of $B$ on $A_{p}(G)$. In fact, both $B$ and $B \times B$ act on $L^{p}(G) \hat{\otimes} L^{p^{\prime}}(G)$. For $f \in L^{p}(G), g \in L^{p^{\prime}}(G)$, and $\beta, \beta^{\prime} \in B$ set

$$
\begin{gathered}
\beta(f \otimes g):=\left({ }^{\beta} f\right) \otimes\left({ }^{\beta} g\right) ; \\
\left(\beta, \beta^{\prime}\right)(f \otimes g):=\left({ }^{\beta} f\right) \otimes\left({ }^{\beta^{\prime}} g\right) .
\end{gathered}
$$

Equation (2.7) (resp. (2.8)) defines a strongly continuous representation of $B$ (resp. $B \times B)$ on $L^{p}(G) \hat{\otimes} L^{p^{\prime}}(G)$, acting as isometries. We need only remark that

$$
\begin{aligned}
\left\|f \otimes g-{ }^{\beta}(f \otimes g)\right\| & =\left\|f \otimes g-\left({ }^{\beta} f\right) \otimes g+\left({ }^{\beta} f\right) \otimes g-\left({ }^{\beta} f\right) \otimes\left({ }^{\beta} g\right)\right\| \\
& \leq\left\|f-{ }^{\beta} f\right\|_{p}\|g\|_{p^{\prime}}+\|f\|_{p}\left\|g-{ }^{\beta} g\right\|_{p^{\prime}} .
\end{aligned}
$$

From equation (1.1) we see that if $h \in L^{p}(G) \hat{\otimes} L^{p^{\prime}}(G)$ and if $\beta \in B$ then

$$
P\left({ }^{\beta} h\right)={ }^{\beta}(P h) \text {. }
$$

2.10 LEMMA. If $f \in A_{p}(G)$ and $\beta \in B$ then ${ }^{\beta} f \in A_{p}(G)$ and the map $\beta \mapsto$ ${ }^{\beta} f$ is a strongly continuous representation of $B$ on $A_{p}(G)$, acting by isometries. Furthermore, $Z_{B} f \in A_{p}(G)$ and $\left\|Z_{B} f\right\|_{A_{p}(G)} \leq\|f\|_{A_{p}(G)}$.

The case $p=2$ was proved in $[\mathrm{PtSu}]$. The following results were verified by Mosak [Msk, p. 284].

2.11 LEMMA. (i) If $f \in L^{1}(G)$ and $g \in{ }^{B} L^{1}(G)$ then $Z_{B}(f * g)=\left(Z_{B} f\right) * g$ and $Z_{B}(g * f)=g *\left(Z_{B} f\right)$.

(ii) If $f, h \in L^{1}(G)$ then $Z_{B}(f * h)=Z_{B}(h * f)$.

(iii) If $1<p<\infty, f \in L^{p}(G)$ and $g \in L^{p^{\prime}}(G)$ then $Z_{B}(f * g)=Z_{B}(g * f)$.

2.12 CoROllary. If $1<p<\infty$ then ${ }^{B} A_{p}(G)={ }^{B} A_{p^{\prime}}(G)$ with equality of norms.

Note that ${ }^{B} A_{p}(G)$ is a closed subalgebra of $A_{p}(G)$. If $h \in{ }^{B} A_{p}(G)$ and $\beta \in B$ then $(M h)(\beta(x), \beta(y))=h\left(\beta\left(x y^{-1}\right)\right)=M h(x, y)$, so that $M h$ is a multiplier of the invariant subspace

$$
{ }^{B}\left(L^{p}(G) \otimes L^{p^{\prime}}(G)\right)
$$

The action of $B \times B$ does not fit in with $P$, for if $f \in L^{p}(G), g \in L^{p^{\prime}}(G)$, and $\beta, \beta^{\prime} \in B$ then

$$
P\left({ }^{\beta} f \otimes \beta^{\beta^{\prime}} g\right)=\left({ }^{\beta^{\prime}} g\right) *\left({ }^{\beta} f\right)^{\vee}={ }^{\beta} P\left(f \otimes{ }^{\beta^{-1} \cdot \beta^{\prime}} g\right) .
$$


In fact, $F \in L^{p}(G) \hat{\otimes} L^{p^{\prime}}(G)$ is $B$-invariant if and only if

$$
F=\int_{B}\left({ }^{\beta} F\right) d m_{B}(\beta), \quad \text { (Bochner integral) }
$$

while it is $B \times B$-invariant if and only if it belongs to $\left({ }^{B} L^{p}(G)\right) \hat{\otimes}\left({ }^{B} L^{p^{\prime}}(G)\right)$. Eymard's question [Ey, 9.3] asks if

$$
{ }^{B} A_{p}(G)=P\left(\left({ }^{B} L^{p}(G)\right) \hat{\otimes}\left({ }^{B} L^{p^{\prime}}(G)\right)\right) ?
$$

Peters $[\mathbf{P t}]$ has shown that the answer is yes when $p=2$.

Let us use the abbreviation

$$
\mathfrak{A}_{p}(G, B):=P\left(\left({ }^{B} L^{p}(G)\right) \hat{\otimes}\left({ }^{B} L^{p^{\prime}}(G)\right)\right) .
$$

This is the analogue of a Figà-Talamanca-Herz space for the hypergroup of $B$ orbits in $G$ (see [Ha, HHL]).

2.15 REMARK. We cannot use the technique of $\left[\mathrm{Hz}_{2} 2\right]$ to verify whether $\mathfrak{A}_{p}(G, B)$ is an algebra. For if $h \in \mathfrak{A}_{p}(G, B) \subseteq{ }^{B} A_{p}(G)$ then $M h$ is a multiplier of ${ }^{B}\left(L^{p}(G) \hat{\otimes} L^{p^{\prime}}(G)\right)$ but not necessarily of $\left({ }^{B} L^{p}(G)\right) \hat{\otimes}\left({ }^{B} L^{p^{\prime}}(G)\right)$, since it need not be $B \times B$-invariant. The best we can say is that the function

$$
(x, y) \mapsto \int_{B} h\left(x \cdot \beta\left(y^{-1}\right)\right) d m_{B}(\beta)
$$

is a multiplier of $\left({ }^{B} L^{p}(G)\right) \hat{\otimes}\left({ }^{B} L^{p^{\prime}}(G)\right)$.

3. Invariant convolution operators. Maintain the notation and hypotheses of $\S 2$. The compact group $B$ acts on $C v_{p}(G)$ via conjugation. If $T \in C v_{p}(G)$ and $\beta \in B$ let ${ }^{\beta} T$ be the bounded linear transformation on $L^{p}(G)$ defined by

$$
\left({ }^{\beta} T\right) f:={ }^{\beta^{-1}}\left(T\left({ }^{\beta} f\right)\right), \quad \forall f \in L^{p}(G) .
$$

An elementary calculation confirms that ${ }^{\beta} T \in C v_{p}(G)$ and clearly

$$
\left\|{ }^{\beta} T\right\|_{p}=\||| T \mid\|_{p}
$$

3.1 Definition. We set ${ }^{B} C v_{p}(G)=\left\{T \in C v_{p}(G):{ }^{\beta} T=T, \forall \beta \in B\right\}$. From (2.2) and (1.1) it follows that if $\varphi \in A_{p}(G)$ can be written as $P\left(\sum_{n=0}^{\infty} f_{n} \otimes g_{n}\right)$ and if $T \in C v_{p}(G)$ then

$$
\begin{aligned}
\left\langle{ }^{\beta} T, \varphi\right\rangle & =\sum_{n=0}^{\infty} g_{n} *\left({ }^{\beta^{-1}}\left(T\left({ }^{\beta} f_{n}\right)\right)\right)^{\vee}(1) \\
& =\sum_{n=0}^{\infty}\left({ }^{\beta} g_{n}\right) *\left(\left(T\left({ }^{\beta} f_{n}\right)\right)\right)^{\vee}(1) \\
& =\left\langle T,{ }^{\beta} \varphi\right\rangle, \quad \forall \beta \in B .
\end{aligned}
$$

We wish to show that ${ }^{B} C v_{p}(G)$ is the closure of $\lambda\left({ }^{B} C_{c}(G)\right)$.

3.3 LEMMA. If $\varphi \in C_{c}(G)$ then $\left\|\mid \lambda\left(Z_{B} \varphi\right)\right\|\left\|_{p} \leq\right\|\|\lambda(\varphi)\| \|_{p}$.

ProOF. We know that

$$
\left\|\left|\lambda\left(Z_{B} \varphi\right)\|\|_{p}=\sup \right| \int_{G} f\left(Z_{B} \varphi\right) d m_{G} \mid\right.
$$


where the supremum is taken over $\left\{f \in A_{p}(G):\|f\|_{A_{p}} \leq 1\right\}$. However,

$$
\left|\int_{G} f\left(Z_{B} \varphi\right) d m_{G}\right|=\left|\int_{B} \int_{G}\left({ }^{\beta} f\right) \varphi d m_{G} d m_{B}\right|,
$$

since $m_{G}$ is $B$-invariant, and the right-hand side is less than or equal to

$$
\int_{B}\|\| \lambda(\varphi)\|\|_{p}\left\|^{\beta} f\right\|_{A_{p}} d m_{B}
$$

Now apply Lemma 2.10. Q.E.D.

Fix $T \in{ }^{B} C v_{p}(G)$ and let $\left\{h_{\gamma}\right\}_{\gamma} \subset C_{c}(G)$ be a net as described in Lemma 2.4. We have just seen that

$$
\|\| \lambda\left(Z_{B} h_{\gamma}\right)\left|\left\|_{p} \leq\right\|\right| \mid T \|_{p}, \quad \forall \gamma .
$$

Furthermore, for every $\varphi \in A_{p}(G)$,

$$
\begin{aligned}
\langle T, \varphi\rangle & =\int_{B}\left\langle{ }^{\beta} T, \varphi\right\rangle d m_{B}(\beta) \stackrel{(3.2)}{=}\left\langle T, Z_{B} \varphi\right\rangle=\lim _{\gamma}\left(Z_{B} \varphi\right) * h_{\gamma}^{\vee}(1) \\
& \stackrel{(3.4)}{=} \lim _{\gamma} \varphi *\left(Z_{B} h_{\gamma}^{\vee}\right)(1)=\lim _{\gamma}\left\langle\lambda\left(Z_{B} h_{\gamma}\right), \varphi\right\rangle .
\end{aligned}
$$

3.7 LEMMA. If $T \in{ }^{B} C v_{p}(G)$ then there exists a net $\left\{h_{\gamma}\right\} \subset{ }^{B} C_{c}(G)$ such that \|\|$\lambda\left(h_{\gamma}\right)\left\|_{p} \leq\right\||| T \|_{p}, \forall \gamma$, and

$$
\langle T, \varphi\rangle=\lim _{\gamma} \int_{G} \varphi h_{\gamma} d m_{G}, \quad \forall \varphi \in A_{p}(G) .
$$

This shows that ${ }^{B} C v_{p}(G)$ is the image $Z_{B}^{*} C v_{p}(G)$ where $Z_{B}^{*}$ is the adjoint of $Z_{B}: A_{p}(G) \rightarrow A_{p}(G)$. From $\left[\mathbf{M z}\right.$, p. 67], it follows that ${ }^{B} C v_{p}(G) \cong{ }^{B} A_{p}(G)^{*}$.

3.8 Proposition. The dual of the Banach space ${ }^{B} A_{p}(G)$ is equal to ${ }^{B} C v_{p}(G)$, with the pairing as in (2.2).

3.9 Corollary. For $1<p<\infty$ and $G_{1} \in[F I A]_{B}^{-}$we have ${ }^{B} C v_{p}(G)=$ ${ }^{B} C v_{p^{\prime}}(G)$ and ${ }^{B} C v_{p}(G) \subset{ }^{B} C v_{2}(G)$.

PROOF. The first part follows from Corollary 2.12 and the second form from the Riesz-Thorin convexity theorem. Q.E.D.

This is different from the case of all of $C v_{p}(G)$, for there are examples $[\mathbf{H z} \mathbf{4}$, Hz 5, Lh, Ob] of values $p$ and groups $G$ with $C v_{p}(G) \neq C v_{p^{\prime}}(G)$. Corollary 3.9 is very well known for various special cases, such as locally compact abelian groups and compact groups, with $B$ the group of inner automorphisms.

3.10 REMARKS. Recall the notation of $\S 1$. Hartmann [Ha] has shown that

$$
{ }^{B} A_{2}(G)=\mathfrak{A}_{2}(G, B) \cong L^{1}\left(\mathfrak{X}_{B}, \nu\right),
$$

where the isomorphism is the "inverse Fourier transform" $\mathcal{F}^{-1}$. Hence, ${ }^{B} C v_{2}(G) \cong$ $L^{\infty}\left(\mathfrak{X}_{B}, \nu\right)$, so that elements of ${ }^{B} C v_{2}(G)$ can be viewed as multipliers. That is, if $T \in{ }^{B} C v_{2}(G)$ then there is $\mathcal{F} T \in L^{\infty}\left(\mathfrak{X}_{B}, \nu\right)$ such that

$$
\langle T, \varphi\rangle=\int_{\mathfrak{X}_{B}}(\mp T)(\mp \varphi) d \nu,
$$

for all $\varphi \in{ }^{B} A_{2}(G) \cap C_{c}(G)$. 
Conversely, $m \in L^{\infty}\left(\mathfrak{X}_{B}, \nu\right)$ is equal to $₹ T$ for some $T \in{ }^{B} C v_{p}(G)$ if and only if

$$
\left|\int_{\mathfrak{X}_{B}} m \cdot \Im \varphi \cdot d \nu\right| \leq \text { const } \cdot\|\varphi\|_{A_{p}(G)}
$$

for all $\varphi \in{ }^{B} A_{p}(G) \cap C_{c}(G)$. We could also use ${ }^{B} A_{2}(G) \cap C_{c}(G)$, equipped with $\|\cdot\|_{A_{p}(G)}$, as a test space in (3.11). See $[\mathbf{C w}]$.

This line of reasoning suggests a means of sometimes distinguishing $A_{p}(G)$ and $\mathfrak{A}_{p}(G, B)$.

Observe that ${ }^{B} L^{1}(G)$ acts on ${ }^{B} L^{p}(G)$ via convolution. Let us denote by $N_{p}(f)$ the norm of $\lambda(f):{ }^{B} L^{p}(G) \rightarrow{ }^{B} L^{p}(G)$, where $f \in{ }^{B} L^{1}(G)$. Clearly, from (2.14) we know that

$$
N_{p}(f)=\sup \left\{\left|\int_{G} f \varphi d m_{G}\right|: \varphi \in \mathfrak{A}_{p}(G, B),\|\varphi\|_{\mathfrak{A}_{p}} \leq 1\right\} .
$$

If one could show that $N_{p}(f) \neq\|\| \lambda(f)\|\|_{p}$, for some $f \in{ }^{B} L^{1}(G)$, then it would follow that $\mathfrak{A}_{p}(G, B) \neq{ }^{B} A_{p}(G)$, since

$$
\|\mid \lambda(f)\|_{p}=\sup \left\{\left|\int_{G} f \varphi d m_{G}\right|: \varphi \in{ }^{B} A_{p}(G),\|\varphi\|_{A_{p}} \leq 1\right\} .
$$

We shall demonstrate this for special cases in the next sections.

4. Radial multipliers. In this section we let $G=\mathbf{R}^{n}$, for fixed $n>1$, and $B=S O(n)$, so that ${ }^{B} L^{p}(G)$ is the subspace of radial elements of $L^{p}\left(\mathbf{R}^{n}\right)$. We use $\hat{f}$ to denote the usual Fourier transform of an integrable function $f$ on $\mathbf{R}^{n}$. The Schwartz space is denoted by $S\left(\mathbf{R}^{n}\right)$ and $D\left(\mathbf{R}^{n}\right)$ is the space of $C^{\infty}$-functions with compact support.

It is well known that $T \in{ }^{S O(n)} C v_{p}\left(\mathbf{R}^{n}\right)$ corresponds to an element $₹ T \in$ $L^{\infty}([0, \infty))$ such that

$$
(T f)(x)=\int_{\mathbf{R}^{n}} \hat{f}(\xi) \mp T(|\xi|) e^{i x \cdot \xi} d \xi,
$$

for all $f \in S\left(\mathbf{R}^{n}\right)$. Conversely, we have seen in $\S 3$ that $m \in L^{\infty}([0, \infty))$ is of the form $m=\mathcal{F} T$, for some $T \in{ }^{S O(n)} C v_{p}\left(\mathbf{R}^{n}\right)$, provided

$$
\left|\int_{\mathbf{R}^{n}} \hat{f}(\xi) m(|\xi|) d \xi\right| \leq \text { const. }\|f\|_{A_{p}\left(\mathbf{R}^{n}\right)}
$$

for all $f \in \in^{S O(n)} D\left(\mathbf{R}^{n}\right)$.

For each $r>0$ let $T_{r}^{\circ}$ be the operator defined by

$$
\left(T_{r}^{\circ} f\right)(x)=\int_{|\xi| \leq r} \hat{f}(\xi) e^{i \xi \cdot x} d \xi
$$

for all $f \in S\left(\mathbf{R}^{n}\right)$. We recall the following results of Herz $\left[\begin{array}{ll}H z & 1\end{array}\right]$ and Fefferman [Ff].

4.2 Lemma. (a) For $n>0, r>0$, and $2 n /(n+1)<p \leq 2$ the operator $T_{r}^{\circ}$ is bounded on ${ }^{S O(n)} L^{p}\left(\mathbf{R}^{n}\right)$ and the norm is independent of $r$.

(b) For $n>1, r>0$, and $p \neq 2, T_{r}^{\circ} \notin C v_{p}\left(\mathbf{R}^{n}\right)$.

The following lemma was shown to me by Michael Cowling. 
4.3 LEMMA. Let $\psi \in C^{\infty}([0, \infty))$ be compactly supported and have $\psi^{\prime} \leq 0$. Furthermore let $T_{\psi}$ be the operator defined by

$$
\left(T_{\psi} f\right)^{\wedge}(\xi)=\psi(|\xi|) \hat{f}(\xi), \quad \forall \xi \in \mathbf{R}^{n}, f \in S\left(\mathbf{R}^{n}\right) .
$$

Then for each $2 n /(n+1)<p<2$ there is a constant $c_{p}>0$ such that

$$
\left\|T_{\psi} f\right\|_{p} \leq c_{p}\|f\|_{p} \psi(0), \quad \text { for all } f \in{ }^{S O(n)} S\left(\mathbf{R}^{n}\right) .
$$

ProOF. For $f, g \in{ }^{S O(n)} S\left(\mathbf{R}^{n}\right)$ we see that

$$
\left(T_{\psi} f\right) * g(0)=\int_{\mathbf{R}^{n}} \hat{f}(\xi) \hat{g}(\xi) \psi(|\xi|) d \xi
$$

Integrating by parts we see that this is equal to

$$
-\int_{0}^{\infty} \psi^{\prime}(r) \int_{|\xi| \leq r} \hat{f}(\xi) \hat{g}(\xi) d \xi d r=-\int_{0}^{\infty} \psi^{\prime}(r)\left(\left(T_{r}^{\circ} f\right) * g\right)(0) d r
$$

Now apply the preceding lemma. Q.E.D.

Note that we could also have used [GT, p. 238] and [Ig].

We can now give a partial answer to $[\mathbf{E y}, 9.3]$.

4.4 Theorem. For $n>1$ and $2 n /(n+1)<p<2$,

$$
{ }^{S O(n)} A_{p}\left(\mathbf{R}^{n}\right) \neq P\left({ }^{S O(n)} L^{p}\left(\mathbf{R}^{n}\right) \hat{\otimes} S O(n) L^{p^{\prime}}\left(\mathbf{R}^{n}\right)\right) .
$$

PROOF. Suppose ${ }^{S O(n)} A_{p}\left(\mathbf{R}^{n}\right)=\mathfrak{A}_{p}\left(\mathbf{R}^{n}, S O(n)\right)$. The open mapping theorem implies equivalence of norms

$$
\|f\|_{A_{p}} \leq\|f\|_{\boldsymbol{A}_{p}} \leq \kappa\|f\|_{A_{p}} .
$$

Fix a smooth, compactly supported function $\psi$ on $[0, \infty]$ such that:

(i) $\psi(t)=1$ if $t \leq 1$;

(ii) $0 \leq \psi(t)<1$ if $t>1$; and

(iii) $\psi^{\prime}(t) \leq 0, \forall t \geq 0$.

For each $k \geq 1$ let $\Psi_{k}$ be the element of $S O(n) S(\mathbf{R})$ such that

$$
\hat{\mathbf{\Psi}}_{k}(\xi)=(\psi(|\xi|))^{k}, \quad \forall \xi \in \mathbf{R}^{n} .
$$

For an arbitrary pair $f, g \in D\left(\mathbf{R}^{n}\right)$ our hypothesis implies that there exists sequences $\left\{F_{l}\right\}_{l \geq 0}$ and $\left\{G_{l}\right\}_{l \geq 0}$ contained in $S O(n) D\left(\mathbf{R}^{n}\right)$ and satisfying

$$
Z_{S O(n)}(f * g)=\sum_{l=0}^{\infty} F_{l} * G_{l}
$$

and $\sum_{l=0}^{\infty}\left\|F_{l}\right\|_{p}\left\|G_{l}\right\|_{p^{\prime}} \leq 2 \kappa\|f\|_{p}\|g\|_{p^{\prime}}$. This involves Lemma 2.10 and the density of $S O(n) D\left(\mathbf{R}^{n}\right)$ in $S O(n) L^{p}\left(\mathbf{R}^{n}\right)$. We now examine

$$
\begin{aligned}
\left|\left\langle\lambda\left(\Psi_{k}\right), f * g\right\rangle\right| & =\left|\left\langle\lambda\left(\Psi_{k}\right), Z_{S O(n)}(f * g)\right\rangle\right| \\
& =\left|\sum_{l=0}^{\infty} \Psi_{k} * F_{l} * G_{l}(0)\right| \leq \sum_{l=0}^{\infty} c_{p}\left\|F_{l}\right\|_{p}\left\|G_{l}\right\|_{p^{\prime}}
\end{aligned}
$$

on account of Lemma 4.3.

However, this shows that for all $k \geq 1$,

$$
\left|\int_{\mathbf{R}^{n}} \hat{f}(\xi) \hat{g}(f)(\psi(|\xi|))^{k} d \xi\right| \leq 2 \kappa c_{p}\|f\|_{p}\|g\|_{p^{\prime}}
$$

The left-hand side converges to $\left|\left\langle T_{1}^{\circ}, f * g\right\rangle\right|$ as $k \rightarrow \infty$ and so (4.5) contradicts Fefferman's solution of the multiplier problem for the ball, [Ff]. Q.E.D. 
5. Central multipliers. Let $G$ be a $d$-dimensional, compact, simply connected, simple Lie group of rank $r$, with a fixed maximal torus $T$. In this case $G \in[F I A]_{B}^{-}$, with $B$ the group of inner automorphisms of $G$, and $Z_{B}$ is the operation of centralization,

$$
Z_{B} f(x)=\int_{G} f\left(y x y^{-1}\right) d m_{G}(y) .
$$

Hence, ${ }^{B} A_{p}(G)$ is the subalgebra of central functions in $A_{p}(G)$ and ${ }^{B} L^{p}(G)$ is the subspace of central elements of $L^{p}(G)$.

We use some results of Stanton and Tomas, [SnTo], to show that ${ }^{B} A_{p}(G) \neq$ $\mathfrak{A}_{p}(G, B)$ for certain values of $p$. Fix a Weyl group-invariant polyhedron $R$ in the Lie algebra of $T$ and let $\left\{D_{n}: n \geq 1\right\}$ be the Dirichlet kernels for summation of Fourier series on $G$, as described in [SnTo, p. 478]. There is a constant $p(R)$, satisfying

$$
2 d /(d+r) \leq p(R) \leq(2 d-2 r+2) /(d-r+2)<2,
$$

such that for all $p(R)<p \leq 2$ and $n \geq 1$

$$
\left\|D_{n} * f\right\|_{p} \leq \text { const. } p\|f\|_{p}, \quad \forall f \in{ }^{B} L^{p}(G) .
$$

However, if $p \neq 2$ then

$$
\sup _{n \geq 1}\left\|\lambda\left(D_{n}\right)\right\|_{p}=\infty
$$

5.1 TheOREM. For $G, B, R$ and $p(R)$ as above and $p(R)<p<2$, we have

$$
{ }^{B} A_{p}(G) \neq P\left({ }^{B} L^{p}(G) \hat{\otimes}^{B} L^{p^{\prime}}(G)\right) .
$$

This is an immediate consequence of $\S 3$ and [SnTo, Theorems D and E].

ACKNOWLEDGEMENTS. This paper would not have reached its final form without the encouragement and valuable comments of Michael Cowling. I am particularly grateful for his proof of Lemma 4.3. While working on this material I have been supported by the Universities of New South Wales and Adelaide. Special thanks to Jenny, Zena and Ruth.

\section{REFERENCES}

[Br] Jean Braconnier, Sur les groupes topologiques localement compacts, J. Math. Pures Appl. 27 (1948), 1-85.

[Cw] Michael Cowling, Some applications of Grothendieck's theory of topological tensor products in harmonic analysis, Math. Ann. 232 (1978), 273-285.

[Ey] Pierre Eymard, Algèbres $A_{p}$ et convoluteurs de $L^{p}$, Sèm. Bourbaki 367, Nov. 1969.

[Ff] Charles Fefferman, The multiplier problem for the ball, Ann. of Math. 94 (1971), 330-336.

[FT] Alessandro Figà-Talamanca, Translation invariant operators in $L^{p}$, Duke Math. J. 32 (1965), 495-502.

[GT] George Gasper and Walter Trebels, Multiplier criteria of Hörmander type for Fourier series and applications to Jacobi series and Hankel transforms, Math. Ann. 242 (1979), 225-240.

[Go] Roger Godement, Introduction aux travaux de A. Selberg, Sém. Bourbaki 144, 1957.

[Ha] Klaus Hartmann, $[F I A]_{B}^{-}$Gruppen und Hypergruppen, Monatsh. Math. 89 (1980), 9-17.

[HHL] Klaus Hartmann, Rolf Wim Henrichs and Rupert Lasser, Duals of orbit spaces in groups with relatively compact inner automorphism groups are hypergroups, Monatsh. Math. 88 (1979), 229-238.

[Hz 1] Carl S. Herz, On the mean inversion of Fourier and Hankel transforms, Proc. Nat. Acad. Sci. U.S.A. 40 (1954), 996-999.

[Hz 2] _ The theory of $p$-spaces with an application to convolution operators, Trans. Amer. Math. Soc. 154 (1971), 69-82. 
[Hz 3] _ Harmonic synthesis for subgroups, Ann. Inst. Fourier (Grenoble) 23 (1973), 91-123.

$\left[\begin{array}{ll}\mathbf{H z} & 4\end{array}\right]$, On the asymmetry of norms of convolution operators. I, J. Funct. Anal. 23 (1976), 11-22.

[Hz 5] _ Asymmetry of norms of convolution operators. II: Nilpotent Lie groups, Symposia Math. 22 (1977), 223-230.

[HwRs] Edwin Hewitt and Kenneth A. Ross, Abstract harmonic analysis. I and II, Springer-Verlag, Berlin, Heidelberg and New York, 1963 and 1970.

[Ig] Satoru Igari, On the multipliers of Hankel transform, Tôhoku Math. J. 24 (1972), 201-206.

[KS] Eberhard Kaniuth and Detlef Steiner, On complete regularity of group algebras, Math. Ann. 204 (1973), 305-329.

[LM] J. Liukkonen and R. Mosak, Harmonic analysis and centers of group algebras, Trans. Amer. Math. Soc. 195 (1974), 147-163.

[Lh] Noël Lohoué, Estimations $L^{p}$ des coefficients de représentation et opérateurs de convolution, Adv. in Math. 38 (1980), 178-221.

[Mz] Michel Mizony, Contribution a l'analyse harmonique sphérique, Publ. Dép. Math. (Lyon) 12-1 (1975), 61-108.

[Msk] Richard D. Mosak, The $L^{1}$ and $C^{*}$-algebras of $[F I A]_{B}^{-}$groups, and their representations, Trans. Amer. Math. Soc. 163 (1972), 277-310.

[Ob] Daniel M. Oberlin, $M_{p}(G) \neq M_{q}(G)\left(p^{-1}+q^{-1}=1\right)$, Israel J. Math. $22(1975), 175179$.

[Pa] T. W. Palmer, Classes of nonabelian, noncompact, locally compact groups, Rocky Mountain J. Math. 8 (1978), 683-741.

[Pt] Justin Peters, Representing positive definite $B$ invariant functions on $[F C]_{B}$ groups, Monatsh. Math. 80 (1975), 319-324.

[PtSu] Justin Peters and Terje Sund, Automorphisms of locally compact groups, Pacific J. Math. 76 (1978), 143-156.

[Rb] Stephen G. Roberts, $A_{p}$ spaces and asymmetry of $L_{p}$-operator norms for convolution operators, M.Sc. Thesis, Flinders University of South Australia, 1982.

[SnTo] Robert J. Stanton and Peter A. Tomas, Polyhedral summability of Fourier series on compact Lie groups, Amer. J. Math. 100 (1978), 477-493.

Department of Pure Mathematics, University of Adelaide, G.P.O. Box 498 , Adelaide, South Australia 5001, Australia

Current address: Department of Mathematics, University of Texas, Austin, Texas 78712 